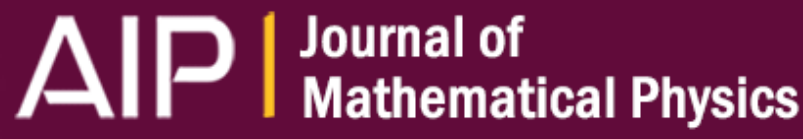

A note on a strongly damped wave equation with fast growing nonlinearities

Varga Kalantarov and Sergey Zelik

Citation: Journal of Mathematical Physics 56, 011501 (2015); doi: 10.1063/1.4905234

View online: http://dx.doi.org/10.1063/1.4905234

View Table of Contents: http://scitation.aip.org/content/aip/journal/jmp/56/1?ver=pdfcov

Published by the AIP Publishing

\section{Articles you may be interested in}

Local well-posedness for nonlinear Klein-Gordon equation with weak and strong damping terms AIP Conf. Proc. 1479, 2155 (2012); 10.1063/1.4756618

Blow up of a solution for a system of nonlinear higher-orderwave equations with strong damping terms

AIP Conf. Proc. 1470, 203 (2012); 10.1063/1.4747675

Periodical Solutions of Certain Strongly Nonlinear Wave Equations

AIP Conf. Proc. 1389, 442 (2011); 10.1063/1.3636758

Nonlinear Hamiltonian equations with fractional damping

J. Math. Phys. 41, 2135 (2000); 10.1063/1.533231

On dimension of the global attractor for damped nonlinear wave equations

J. Math. Phys. 40, 1432 (1999); 10.1063/1.532813

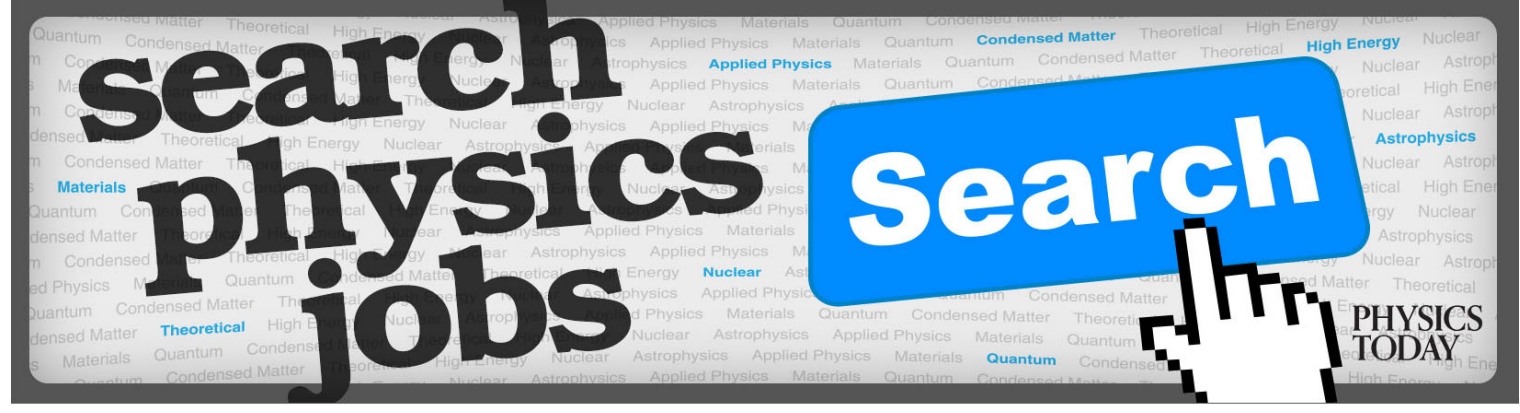




\title{
A note on a strongly damped wave equation with fast growing nonlinearities
}

\author{
Varga Kalantarov ${ }^{1, a)}$ and Sergey Zelik 2, b) \\ ${ }^{1}$ Department of Mathematics, Koç University, Rumelifeneri Yolu, \\ Sariyer, Istanbul 34450, Turkey \\ ${ }^{2}$ University of Surrey, Department of Mathematics, Guildford GU2 7XH, United Kingdom
}

(Received 28 July 2014; accepted 17 December 2014; published online 9 January 2015)

\begin{abstract}
A strongly damped wave equation including the displacement depending nonlinear damping term and nonlinear interaction function is considered. The main aim of the note is to show that under the standard dissipativity restrictions on the nonlinearities involved, the initial boundary value problem for the considered equation is globally well-posed in the class of sufficiently regular solutions and the semigroup generated by the problem possesses a global attractor in the corresponding phase space. These results are obtained for the nonlinearities of an arbitrary polynomial growth and without the assumption that the considered problem has a global Lyapunov function. (C) 2015 AIP Publishing LLC. [http://dx.doi.org/10.1063/1.4905234]
\end{abstract}

\section{INTRODUCTION}

In a bounded smooth domain $\Omega \subset \mathbb{R}^{3}$, we consider the following problem:

$$
\left\{\begin{array}{l}
\partial_{t}^{2} u+f(u) \partial_{t} u-\gamma \partial_{t} \Delta_{x} u-\Delta_{x} u+g(u)=h,\left.u\right|_{\partial \Omega}=0, \\
\left.u\right|_{t=0}=u_{0},\left.\partial_{t} u\right|_{t=0}=u_{1} .
\end{array}\right.
$$

Here, $u=u(t, x)$ is unknown function, $\Delta_{x}$ is the Laplacian with respect to the variable $x, \gamma>0$ is a given dissipation parameter, $f$ and $g$ are given nonlinearities, and $h \in L^{2}(\Omega)$ are given external forces. We assume throughout of the paper that the nonlinearities $f$ and $g$ satisfy

$$
\left\{\begin{array}{l}
\text { 1. } f, g \in C^{1}(R), \\
\text { 2. }-C+\alpha|u|^{p} \leq f(u) \leq C_{1}\left(1+|u|^{p}\right), \\
\text { 3. }-C+\alpha|u|^{q} \leq g^{\prime}(u) \leq C\left(1+|u|^{q}\right),
\end{array}\right.
$$

where $p, q \geq 0$ and $p+q>0$.

Strongly damped wave equations of the form (1.1) and similar equations are of a great current interest, see Refs. 1, 5-9, 13, 15, 17-19, 21, 23, 26, 27 and references therein. The most studied version of the equation (1.1) is the case with only one nonlinearity $(f \equiv 0)$, i.e., the problem of the form

$$
\left\{\begin{array}{l}
\partial_{t}^{2} u-\gamma \partial_{t} \Delta_{x} u-\Delta_{x} u+g(u)=h,\left.u\right|_{\partial \Omega}=0, \\
\left.u\right|_{t=0}=u_{0},\left.\partial_{t} u\right|_{t=0}=u_{1} .
\end{array}\right.
$$

Even in this particular case, the equation has a lot of non-trivial and interesting features attracting the attention of many mathematicians, see Refs. 5, 6, 8, 9, 17, 18, 27, 28 and references therein. For instance, it has been thought for a long time that there is a critical growth exponent $q_{\max }=4$ for the nonlinearity $g$ and that the properties of the solutions in the supercritical case $q>q_{\max }$ are principally different from the subcritical case $q<q_{\max }$. However, it was shown in Ref. 27 that

\footnotetext{
a) E-mail address: vkalantarov@ku.edu.tr

b) E-mail address: s.zelik@surrey.ac.uk
} 
problem (1.3) with nonlinear term satisfying just the condition $g^{\prime}(s) \geq-C, \forall s \in \mathbb{R}$ has a global unique solution belonging to the phase space

$$
\mathcal{E}_{1}:=\left[H^{2}(\Omega) \cap H_{0}^{1}(\Omega)\right] \times H_{0}^{1}(\Omega), \quad \xi_{u}(t) \in \mathcal{E}_{1}, t \geq 0 .
$$

So it was established that there is no critical exponent at least in the class of smooth solutions $\xi_{u}:=\left(u, \partial_{t} u\right)$. A dissipativity of the semigroup generated by problem (1.3) in the phase space $\mathcal{E}_{1}$ was shown in Refs. 10 and 22; in Ref. 22, regularity of the attractor of the semigroup was also established.

Here and below, $H^{s}(\Omega)$ stands for the usual Sobolev space of distributions whose derivatives up to order $s$ belong to $L^{2}(\Omega)$ and $H_{0}^{s}(\Omega)$ means the closure of $C_{0}^{\infty}(\Omega)$ in $H^{s}(\Omega)$.

In what follows to simplify notations, we will denote by $C$ various constants that do not depend on the initial data.

The global unique solvability of the initial boundary value problem (1.3) with $g(u)$ satisfying just condition (1.2) with arbitrary $q \in \mathbb{R}^{+}$has been established later on for the case of solutions belonging to the natural energy space

$$
\mathcal{E}_{f=0}:=\left[H_{0}^{1}(\Omega) \cap L^{q+2}(\Omega)\right] \times L^{2}(\Omega) .
$$

Thus, it was shown that even on the level of energy solutions, there is no critical exponent for the growth rate of $g$, and despite the expectations, the analytic and dynamic properties (existence and uniqueness, dissipativity, asymptotic smoothing, attractors, and their dimension) look very similar for the cases $q<4$ and $q>4$. This is related with the non-trivial monotonicity properties of the equation considered in the space $L^{2}(\Omega) \times H^{-1}(\Omega)$, see Ref. 11 for more details.

The alternative case when another nonlinearity vanishes, $g \equiv 0$ also leads to essential simplifications. Indeed, assuming that $h=0$ for simplicity and introducing the new variable $v(t):=$ $\int_{0}^{t} u(s) d s$, we reduce (1.1) to

$$
\partial_{t}^{2} v-\gamma \partial_{t} v+F\left(\partial_{t} v\right)-\Delta_{x} v=c
$$

where $c$ depends on the initial data and $F(u):=\int_{0}^{u} f(v) d v$. Using, e.g., the methods of Ref. 12, one can show the absence of a critical exponent for the growth rate of $f(u)$ in the energy phase space.

The situation becomes more complicated when both nonlinearities $f$ and $g$ are presented in the equation and grow sufficiently fast since the methods developed to treat the case of fast growing $g$ are hardly compatible with the methods for $f$ and vise versa. In particular, the problem of presence or absence of critical growth exponents for the non-linearities $f$ and $g$ is still open here and, to the best of our knowledge, a more or less complete theory for this equation (including existence and uniqueness, dissipativity, asymptotic regularity, and attractors) is built up only for the case where $f$ and $g$ satisfy the growth restrictions $p \leq 4, q \leq 4$ and additional monotonicity restriction

$$
f(u) \geq 0, u \in \mathbb{R},
$$

see Ref. 14 for the details.

The main aim of these notes is to show that problem (1.1) is globally well-posed and dissipative at least in the class of the so-called strong solutions $\xi_{u} \in \mathcal{E}_{1}$ without any restrictions on the growth exponents $p$ and $q$ and without the monotonicity assumption (1.6). To be more precise, the main result of the notes is the following theorem.

Theorem 1.1. Let $h \in L^{2}(\Omega)$ and the nonlinearities $f$ and $g$ satisfy assumptions (1.2). Then, for every $\xi_{u}(0) \in \mathcal{E}_{1}$, there exists a unique strong solution $\xi_{u} \in C\left(\mathbb{R}_{+}, \mathcal{E}_{1}\right)$ of (1.1) and the following estimate holds:

$$
\left\|\xi_{u}(t)\right\|_{\mathcal{E}_{1}} \leq Q\left(\left\|\xi_{u}(0)\right\|_{\mathcal{E}_{1}}\right) e^{-\alpha t}+Q\left(\|h\|_{L^{2}}\right)
$$

for some positive constant $\alpha$ and monotone function $Q$, where

$$
\left\|\xi_{u}(t)\right\|_{\mathcal{E}_{1}}^{2}:=\left\|\nabla_{x} \partial_{t} u(t)\right\|^{2}+\left\|\Delta_{x} u(t)\right\|^{2} .
$$

The proof of this theorem is given in Sec. II. 
The dissipative estimate (1.7) is strong enough to obtain the existence of a global attractor $\mathcal{A}$ for the considered system in the phase space $\mathcal{E}_{1}$ and verify that its smoothness is restricted by the regularity of the data $f, g$, and $h$ only, see Sec. III for more details. Note also that, in contrast to the most part of papers on the subject, we do not use the monotonicity assumption (1.6). As a result, the equation does not possess any more a global Lyapunov function and the non-trivial dynamics on the attractor becomes possible. For instance, our assumptions include the Van der Pole nonlinearities $f(u)=u^{3}-u$ and $g(u)=u$, so the time periodic orbits (and chaotic dynamics) become possible. Another classical example with non-trivial dynamics is the so-called FitzHugh-Nagumo system

$$
\left\{\begin{array}{l}
\partial_{t} u=\Delta_{x} u-\phi(u)-v, \\
\partial_{t} v=u-v .
\end{array}\right.
$$

Indeed, differentiating the first equation by $t$ and removing the variable $v$ using the second equation, we obtain the equation

$$
\partial_{t}^{2} u-\partial_{t} \Delta_{x} u+\psi^{\prime}(u) \partial_{t} u-\Delta_{x} u+\psi(u)-2 u=0,
$$

where $\psi(u)=u+\phi(u)$. So the FitzHugh-Nagumo is indeed a particular case of the strongly damped wave equation of the form (1.1). One more interesting example is related with the so-called Josephson junction arising between two layers of superconductors separated by the insulator layer. Indeed, using the expression for the Josephson tunnel current $J$ involving the $\cos \phi$ term

$$
J(\phi)=j_{0} \sin \phi+j_{1}(1+\varepsilon \cos \phi) \phi_{t},
$$

where $j_{0}, j_{2}, \varepsilon$ are given constants and $\phi$ is the phase difference of the superconducting order parameters, see Ref. 20 and arguing as in Ref. 16, we end up with the strongly damped wave equation for $\phi$

$$
\phi_{t t}-\alpha \phi_{t x x}-\beta(1+\varepsilon \cos \phi) \phi_{t}-\phi_{x x}+\sin \phi=0,
$$

for some parameters $\alpha$ and $\beta$. Note also that the parameter $\varepsilon$ is not necessarily small and the non-monotone case $|\varepsilon|>1$ remains physically relevant, see Ref. 4 .

Thus, relaxing the monotonicity assumption (1.6) indeed makes the theory essentially more general and interesting. As a price to pay, we lose the control over the possible growth of weak energy solutions. Indeed, the energy equality for our problem reads

$$
\frac{d}{d t}\left(\frac{1}{2}\left\|\partial_{t} u\right\|_{L^{2}}^{2}+\frac{1}{2}\left\|\nabla_{x} u\right\|_{L^{2}}^{2}+(G(u), 1)-(h, u)\right)+\gamma\left\|\partial_{t} \nabla_{x} u\right\|_{L^{2}}^{2}+\left(f(u) \partial_{t} u, \partial_{t} u\right)=0
$$

(here and below, $(u, v)$ stands for the classical inner product in $L^{2}(\Omega)$ and $\left.G(u):=\int_{0}^{u} g(v) d v\right)$. We see that under assumptions (1.2), we have only the control $\left(f(u) \partial_{t} u, \partial_{t} u\right) \geq-C\left\|\partial_{t} u\right\|_{L^{2}}^{2}$ which is enough to prove the existence of weak energy solutions but is not sufficient to verify that they are globally bounded in time. Actually, we do not know how to obtain the dissipative energy estimate on the level of weak energy solutions and by this reason have to consider more smooth solutions $\xi_{u} \in \mathcal{E}_{1}$. It worth noting that the presence of the strongly damping term $-\gamma \partial_{t} \Delta_{x} u$ is crucial in obtaining of our main result. The case $\gamma=0$ is more complicated, both monotonicity and growth restrictions seem unavoidable in this case (see, e.g., Refs. 3 and 23).

\section{MAIN ESTIMATE}

The main aim of this section is to prove the key estimate (1.7). We start with slightly weaker dissipative estimate in the space $H^{2}(\Omega) \cap H_{0}^{1}(\Omega) \times L^{2}(\Omega)$.

Theorem 2.1. Let the conditions of Theorem 1.1 be satisfied and let $\xi_{u} \in C\left(\mathbb{R}_{+}, \mathcal{E}_{1}\right)$ be a strong solution of (1.1). Then, the following estimate holds:

$$
\left\|\partial_{t} u(t)\right\|_{L^{2}}^{2}+\|u(t)\|_{H^{2}}^{2}+\int_{t}^{t+1}\left\|\partial_{t} u(s)\right\|_{H^{1}}^{2} d s \leq Q\left(\left\|\partial_{t} u(0)\right\|_{L^{2}}^{2}+\|u(0)\|_{H^{2}}^{2}\right) e^{-\alpha t}+Q\left(\|h\|_{L^{2}}\right),
$$

where the positive constant $\alpha$ and monotone function $Q$ which are independent of $\xi_{u}$. 
Proof. The proof of this estimate is strongly based on the new estimate obtained by multiplication of (1.1) by $v:=\partial_{t} u-\gamma \Delta_{x} u+F(u)$, where $F(u):=\int_{0}^{u} f(v) d v$. It is not difficult to show that under the above assumptions on the solution $\xi_{u}, v \in L^{2}(\Omega)$ and the multiplication is allowed. Then, after the straightforward transformations, we get

$$
\begin{aligned}
\frac{d}{d t}\left(\frac{1}{2}\|v\|_{L^{2}}^{2}+\frac{1}{2}\left\|\nabla_{x} u\right\|_{L^{2}}^{2}+(\right. & G(u), 1))+ \\
& +\gamma\left\|\Delta_{x} u\right\|_{L^{2}}^{2}+\left(f(u)+\gamma g^{\prime}(u),\left|\nabla_{x} u\right|^{2}\right)+(F(u), g(u))=(h, v) .
\end{aligned}
$$

In addition, due to (1.2) and the assumption $p+q>0$,

$$
\begin{aligned}
\left(f(u)+g^{\prime}(u),\left|\nabla_{x} u\right|^{2}\right)+(F(u), g(u)) & \geq \\
& \geq\left(|f(u)|+\left|g^{\prime}(u)\right|,\left|\nabla_{x} u\right|^{2}\right)+\frac{1}{2}(|F(u)|+1,|g(u)|+1)-C\left(\left\|\nabla_{x} u\right\|^{2}+\|u\|^{2}+1\right),
\end{aligned}
$$

and using the interpolation $\left\|\nabla_{x} u\right\|^{2} \leq\left\|\Delta_{x} u\right\|\|u\|$, we have

$$
\begin{aligned}
& \frac{d}{d t}\left(\|v\|_{L^{2}}^{2}+\left\|\nabla_{x} u\right\|_{L^{2}}^{2}+2(G(u), 1)\right)+ \\
& \quad+\gamma\left\|\Delta_{x} u\right\|_{L^{2}}^{2}+\left(|f(u)|+\left|g^{\prime}(u)\right|,\left|\nabla_{x} u\right|^{2}\right)+(|F(u)|+1,|g(u)|+1) \leq C+2\|h\|_{L^{2}}\|v\|_{L^{2}} .
\end{aligned}
$$

This estimate is still not enough to get the desired dissipative estimate since we do not have the positive term related with $\|v\|_{L^{2}}$ without the differentiation.

At the next step, we use the energy equality (1.10) which is obtained by multiplication of (1.1) by $\partial_{t} u$ and which together with our assumptions on $f$ gives

$$
\begin{aligned}
& \frac{d}{d t}\left(\frac{1}{2}\left\|\partial_{t} u\right\|_{L^{2}}^{2}+\frac{1}{2}\left\|\nabla_{x} u\right\|_{L^{2}}^{2}+(G(u), 1)\right)+ \\
& \quad+\left(|f(u)|+1,\left|\partial_{t} u\right|^{2}\right)+\gamma\left\|\partial_{t} \nabla_{x} u\right\|_{L^{2}}^{2} \leq L\left(\left\|\partial_{t} u\right\|_{L^{2}}^{2}+\|h\|_{L^{2}}^{2}\right)
\end{aligned}
$$

for some positive constant $L$.

To estimate the term in the right-hand side of (2.5), we multiply equation (1.1) by $u$ which gives

$$
\left\|\partial_{t} u\right\|_{L^{2}}^{2}=\frac{d}{d t}\left(\left(u, \partial_{t} u\right)+\frac{\gamma}{2}\left\|\nabla_{x} u\right\|_{L^{2}}^{2}\right)+\left\|\nabla_{x} u\right\|_{L^{2}}^{2}+(g(u), u)+\left(f(u) \partial_{t} u, u\right)-(h, u) .
$$

Note that, due to our assumptions (1.2) on the nonlinearity $f$, for any $\beta>0$,

$$
\left|\left(f(u) \partial_{t} u, u\right)\right| \leq \beta\left(|f(u)|,\left|\partial_{t} u\right|^{2}\right)+C_{\beta}\left(|f(u)|+1, u^{2}+1\right) .
$$

Using now the assumption that $p+q>0$, we see that $|f(u)| u^{2} \sim|u|^{p+2}$ and $|F(u) g(u)| \sim|u|^{p+2+q}$ as $u \rightarrow \infty$, therefore

$$
\left|\left(f(u) \partial_{t} u, u\right)\right| \leq \beta\left(|f(u)|,\left|\partial_{t} u\right|^{2}\right)+C_{\beta}(|F(u)|+1,|g(u)|+1)+C_{\beta} .
$$

Inserting this into the right-hand side of (2.5) and fixing $\beta>0$ being small enough, we arrive at

$$
\begin{aligned}
& \frac{d}{d t}\left(\frac{1}{2}\left\|\partial_{t} u\right\|_{L^{2}}^{2}+\frac{1}{2}\left\|\nabla_{x} u\right\|_{L^{2}}^{2}+(G(u), 1)-L\left(\left(u, \partial_{t} u\right)+\frac{\gamma}{2}\left\|\nabla_{x} u\right\|_{L^{2}}^{2}\right)\right)+ \\
& \quad+\frac{1}{2}\left(|f(u)|+1,\left|\partial_{t} u\right|^{2}\right)+\gamma\left\|\partial_{t} \nabla_{x} u\right\|_{L^{2}}^{2} \leq C L(|F(u)|+1,|g(u)|+1)+C\left(\left\|\nabla_{x} u\right\|_{L^{2}}^{2}+\|h\|_{L^{2}}^{2}+1\right) .
\end{aligned}
$$


Taking a sum of (2.9) multiplied by a small parameter $\kappa>0$ with (2.4), we have

$$
\begin{aligned}
\frac{d}{d t}\left(\|v\|_{L^{2}}^{2}+(1+\right. & \left.\frac{\kappa}{2}-L \frac{\kappa \gamma}{2}\right)\left\|\nabla_{x} u\right\|_{L^{2}}^{2}+ \\
& \left.+(2+\kappa)(G(u), 1)-L \kappa\left(u, \partial_{t} u\right)+\frac{\kappa}{2}\left\|\partial_{t} u\right\|_{L^{2}}^{2}\right)+ \\
& +\beta\left(\left\|\partial_{t} u\right\|_{L^{2}}^{2}+\left\|\Delta_{x} u\right\|_{L^{2}}^{2}+\left(|f(u)|+1,\left|\partial_{t} u\right|^{2}\right)+\right. \\
\left.+\left(|f(u)|+\left|g^{\prime}(u)\right|,\left|\nabla_{x} u\right|^{2}\right)+(|F(u)|+1,|g(u)|+1)+\left\|\partial_{t} \nabla_{x} u\right\|_{L^{2}}^{2}\right) \leq & \leq C\left(\|h\|_{L^{2}}\|v\|_{L^{2}}+\|h\|_{L^{2}}^{2}+1\right)
\end{aligned}
$$

for some positive constant $\beta$ depending on $\kappa$. We now note that it is possible to fix $\kappa$ being small enough that the function

$$
\mathcal{E}_{u}(t):=\|v\|_{L^{2}}^{2}+\left(1+\frac{\kappa}{2}-L \frac{\kappa \gamma}{2}\right)\left\|\nabla_{x} u\right\|_{L^{2}}^{2}+(2+\kappa)(G(u), 1)-L \kappa\left(u, \partial_{t} u\right)+\frac{\kappa}{2}\left\|\partial_{t} u\right\|_{L^{2}}^{2}
$$

will satisfy the inequalities

$$
\begin{aligned}
\alpha\left(\|v\|_{L^{2}}^{2}+\left\|\partial_{t} u\right\|_{L^{2}}^{2}+\left\|\nabla_{x} u\right\|_{L^{2}}^{2}+(|G(u)|, 1)\right)-C_{1} \leq \mathcal{E}_{u}(t) \leq \\
\quad \leq C\left(1+\|v\|_{L^{2}}^{2}+\left\|\partial_{t} u\right\|_{L^{2}}^{2}+\left\|\nabla_{x} u\right\|_{L^{2}}^{2}+(|G(u)|, 1)\right)
\end{aligned}
$$

for some positive $\alpha$. Thus, (2.10) reads

$$
\begin{aligned}
\frac{d}{d t} \mathcal{E}_{u}(t)+\beta\left(\left\|\partial_{t} u\right\|_{L^{2}}^{2}+\left\|\Delta_{x} u\right\|_{L^{2}}^{2}+\right. & \left.\left(|f(u)|+1,\left|\nabla_{x} u\right|^{2}\right)+(|F(u)|+1,|g(u)|+1)\right)+ \\
& +\beta\left(|f(u)|+1,\left|\partial_{t} u\right|^{2}\right) \leq C\left(\|h\|_{L^{2}}\|v\|_{L^{2}}+\|h\|_{L^{2}}^{2}+1\right) .
\end{aligned}
$$

Let now $q \geq p$. Then,

$$
(|F(u)|+1)(|g(u)|+1) \sim|u|^{p+q+2} \geq|u|^{2 p+2} \sim F(u)^{2},
$$

and using the obvious estimate

$$
\|v\|_{L^{2}}^{2} \leq C\left(\left\|\partial_{t} u\right\|_{L^{2}}^{2}+\left\|\Delta_{x} u\right\|_{L^{2}}^{2}+\|F(u)\|_{L^{2}}^{2}\right),
$$

we see that, in the case $q \geq p$, (2.13) implies

$$
\frac{d}{d t} \mathcal{E}_{u}(t)+\beta \mathcal{E}_{u}(t) \leq C\left(\|h\|_{L^{2}}^{2}+1\right)
$$

for some positive $\beta$.

It only remains to study the case $p>q$. In this case, we extract the desired $L^{2}$ norm of $F(u)$ from the term $\left(|f(u)|+1,\left|\nabla_{x} u\right|^{2}\right)$. Indeed,

$$
\left(|f(u)|+1,\left|\nabla_{x} u\right|^{2}\right) \geq \alpha\left(|u|^{p},\left|\nabla_{x} u\right|^{2}\right)=\alpha_{1}\left\|\nabla_{x}\left(|u|^{(p+2) / 2}\right)\right\|_{L^{2}}^{2} .
$$

Since $H_{0}^{1}(\Omega)$ is continuously embedded into $L^{4}(\Omega)$, we have

$$
\alpha_{1}\left\|\nabla_{x}\left(|u|^{(p+2) / 2}\right)\right\|_{L^{2}}^{2} \geq \alpha_{3}\left\||u|^{p+2}\right\|_{L^{2}},
$$

and we obtain from (2.16) that

$$
\left(|f(u)|+1,\left|\nabla_{x} u\right|^{2}\right) \geq \alpha_{3}\|F(u)\|_{L^{2}}^{\frac{p+2}{p+1}}-C
$$

for some positive $\alpha_{i}, i=1,2,3$. Thus, (2.13) now reads

$$
\partial_{t} \mathcal{E}_{u}(t)+\beta\left[\mathcal{E}_{u}(t)\right]^{\frac{p+2}{2(p+1)}} \leq C\left(\|h\|_{L^{2}}\left[\mathcal{E}_{u}(t)\right]^{1 / 2}+\|h\|_{L^{2}}^{2}+1\right) .
$$

Since $\frac{p+2}{2(p+1)}>\frac{1}{2}$, the Gronwall type inequality works in both cases and gives the dissipative estimate for $\mathcal{E}_{u}(t)$

$$
\mathcal{E}_{u}(t) \leq Q\left(\mathcal{E}_{u}(0)\right) e^{-\alpha t}+Q\left(\|h\|_{L^{2}}\right)
$$


where the positive constant $\alpha$ and monotone function $Q$ are independent of $t$ and $u$. Note also that, due to the maximal regularity result for the semilinear heat equation,

$$
C\left(\left\|\partial_{t} u(t)\right\|_{L^{2}}^{2}+\|u(t)\|_{H^{2}}^{2}\right)-C \leq\|v(t)\|_{L^{2}}^{2} \leq Q\left(\left\|\partial_{t} u(t)\right\|_{L^{2}}^{2}+\|u(t)\|_{H^{2}}^{2}\right)
$$

for some positive $C$ and $Q$. The desired estimate (1.7) follows in a straightforward way from (2.18) and (2.19). Thus, Theorem 2.1 is proved.

Next proposition gives the uniqueness of the strong solution of equation (1.1).

Proposition 2.2. Let the conditions of Theorem 1.1 hold and let $\xi_{u_{1}}, \xi_{u_{2}} \in C\left(\mathbb{R}_{+}, \mathcal{E}_{1}\right)$ be two solutions of the problem (1.1). Then, the following estimate holds:

$$
\left\|\xi_{u_{1}}(t)-\xi_{u_{2}}(t)\right\|_{\mathcal{E}} \leq C e^{K t}\left\|\xi_{u_{1}}(0)-\xi_{u_{2}}(0)\right\|_{\mathcal{E}},
$$

where the constants $C$ and $K$ depend on the initial data and $\left\|\xi_{u}\right\|_{\mathcal{E}}^{2}:=\left\|\nabla_{x} u\right\|_{L^{2}}^{2}+\left\|\partial_{t} u\right\|_{L^{2}}^{2}$.

Proof. Indeed, let $v:=u_{1}-u_{2}$. Then, this function solves

$$
\partial_{t}^{2} v+f\left(u_{1}\right) \partial_{t} v-\gamma \Delta_{x} v-\Delta_{x} v=-\left[f\left(u_{1}\right)-f\left(u_{2}\right)\right] \partial_{t} u_{2}-\left[g\left(u_{1}\right)-g\left(u_{2}\right)\right] .
$$

Multiplying this equation by $\partial_{t} v$ and using the estimate (2.1) together with the embeddings $H^{2}(\Omega) \subset$ $C(\Omega)$ and $H^{1}(\Omega) \subset L^{4}(\Omega)$, we have

$$
\begin{gathered}
\frac{1}{2} \frac{d}{d t}\left\|\xi_{v}\right\|^{2}+\gamma\left\|\nabla_{x} \partial_{t} v\right\|_{L^{2}}^{2}+\left(f\left(u_{1}\right) \partial_{t} v, \partial_{t} v\right)=- \\
-\left(\left[f\left(u_{1}\right)-f\left(u_{2}\right)\right] \partial_{t} u_{2}, \partial_{t} v\right)-\left(g\left(u_{1}\right)-g\left(u_{2}\right), \partial_{t} v\right) \leq \\
\leq C\|v\|_{L^{2}}\left\|\partial_{t} v\right\|_{L^{4}}\left\|\partial_{t} u_{1}\right\|_{L^{4}}+C\|v\|_{L^{2}}\left\|\partial_{t} v\right\|_{L^{2}} \leq \\
\quad \leq \frac{\gamma}{2}\left\|\nabla_{x} \partial_{t} v\right\|_{L^{2}}^{2}+\left\|\nabla_{x} \partial_{t} u_{1}\right\|_{L^{2}}^{2}\|v\|_{L^{2}}^{2} .
\end{gathered}
$$

Thus, we end up with the following inequality:

$$
\frac{d}{d t}\left\|\xi_{v}\right\|^{2}+\gamma\left\|\nabla_{x} \partial_{t} v\right\|_{L^{2}}^{2} \leq C\left\|\nabla_{x} \partial_{t} u_{2}\right\|_{L^{2}}^{2}\left\|\xi_{v}\right\|_{L^{2}}^{2}
$$

and the Gronwall inequality applied to this relation finishes the proof of the proposition.

We are now ready to check the dissipativity in $\mathcal{E}_{1}$.

Proposition 2.3. Let the conditions of Theorem 1.1 be satisfied. Then, for every $\xi_{u}(0) \in \mathcal{E}_{1}$, there is a unique solution $\xi_{u} \in C\left(\mathbb{R}_{+}, \mathcal{E}_{1}\right)$ of problem (1.1) and the following estimate holds:

$$
\left\|\xi_{u}(t)\right\|_{\mathcal{E}_{1}}+\int_{t}^{t+1}\left\|\partial_{t} u(s)\right\|_{H^{2}}^{2} d s \leq Q\left(\left\|\xi_{u}(0)\right\|_{\mathcal{E}_{1}}\right) e^{-\alpha t}+Q\left(\|h\|_{L^{2}}\right)
$$

for some positive constant $\alpha$ and monotone function $Q$.

Proof. We restrict ourselves to the formal derivation of the dissipative estimate (2.24). The existence of a solution as well as the justification of this derivation can be done in a standard way using, e.g., Galerkin approximations. Moreover, due to (2.1), we only need to obtain the control over the higher norms of the derivative $\partial_{t} u$. To this end, we multiply equation (1.1) by $-\partial_{t} \Delta_{x} u$. Then, after some transformations, we get

$$
\begin{aligned}
\frac{1}{2} \frac{d}{d t}\left\|\xi_{u}\right\|_{\mathcal{E}_{1}}^{2}+\left\|\xi_{u}\right\|_{\mathcal{E}_{1}}^{2}+\gamma\left\|\partial_{t} \Delta_{x} u\right\|_{L^{2}}^{2}=\left(f(u) \partial_{t} u, \partial_{t} \Delta_{x} u\right)+ \\
\quad+\left(g(u), \partial_{t} \Delta_{x} u\right)+\left\|\partial_{t} \nabla_{x} u\right\|_{L^{2}}^{2}+\left\|\Delta_{x} u\right\|_{L^{2}}^{2} \leq C\left(\left\|\partial_{t} u\right\|_{L^{2}}^{2}+\left\|\Delta_{x} u\right\|_{L^{2}}^{2}\right)+\frac{\gamma}{2}\left\|\partial_{t} \Delta_{x} u\right\|_{L^{2}}^{2}
\end{aligned}
$$

where we have implicitly used that $H^{2}(\Omega) \subset C(\Omega)$ and the interpolation $\|v\|_{H^{1}}^{2} \leq C\|v\|_{L^{2}}\|v\|_{H^{2}}$. The obtained estimate gives

$$
\frac{d}{d t}\left\|\xi_{u}\right\|_{\mathcal{E}_{1}}^{2}+\left\|\xi_{u}\right\|_{\mathcal{E}_{1}}^{2}+\gamma\left\|\partial_{t} \Delta_{x} u\right\|_{L^{2}}^{2} \leq C\left(\left\|\partial_{t} u\right\|_{L^{2}}^{2}+\left\|\Delta_{x} u\right\|_{L^{2}}^{2}\right)
$$

and the Gronwall inequality together with (2.1) finishes the proof of the proposition. 


\section{A GLOBAL ATTRACTOR}

In this section, we study the long-time behavior of solutions of problem (1.1) in terms of the associated global attractor. For the reader convenience, we first remind the key definitions of the attractors theory, see Refs. 2 and 25 for more details.

According to Proposition 2.3, the solution operators of problem (1.1) generate a semigroup in the phase space $\mathcal{E}_{1}$

$$
S(t) \xi_{u}(0):=\xi_{u}(t), \quad S(t): \mathcal{E}_{1} \rightarrow \mathcal{E}_{1}, \quad S(t+h)=S(t) \circ S(h), t, h \geq 0 .
$$

Moreover, according to the estimate (2.24), the semigroup $S(t)$ is dissipative in the phase space $\mathcal{E}_{1}$, i.e., the estimate

$$
\|S(t) \xi\|_{\mathcal{E}_{1}} \leq Q\left(\|\xi\|_{\mathcal{E}_{1}}\right) e^{-\alpha t}+Q\left(\|h\|_{L^{2}}\right), \quad \xi \in \mathcal{E}_{1}
$$

holds for some positive constant $\alpha$ and monotone function $Q$.

Definition 3.1. Let $S(t): \mathcal{E}_{1} \rightarrow \mathcal{E}_{1}$ be a semigroup. A set $\mathcal{B} \subset \mathcal{E}_{1}$ is called an attracting set for this semigroup if for every bounded set $B \subset \mathcal{E}_{1}$ and every neighborhood $O(\mathcal{B})$ of the set $\mathcal{B}$, there exists $T=T(B, O)$ such that

$$
S(t) B \subset O(\mathcal{B})
$$

for all $t \geq T$.

Definition 3.2. Let $S(t): \mathcal{E}_{1} \rightarrow \mathcal{E}_{1}$ be a semigroup. A set $\mathcal{A}$ is called a global attractor for the semigroup $S(t)$ if

1. The set $\mathcal{A}$ is compact in $\mathcal{E}_{1}$;

2. The set $\mathcal{A}$ is strictly invariant: $S(t) \mathcal{A}=\mathcal{A}$ for all $t \geq 0$;

3. The set $\mathcal{A}$ is an attracting set for the semigroup $S(t)$.

To verify the existence of a global attractor, we will use the following version of an abstract attractor existence theorem.

Proposition 3.3. Let $S(t): \mathcal{E}_{1} \rightarrow \mathcal{E}_{1}$ be a semigroup satisfying the following two assumptions:

1. There exists a compact attracting set $\mathcal{B}$ for the semigroup $S(t)$.

2. For every $t \geq 0$, the map $S(t): \mathcal{E}_{1} \rightarrow \mathcal{E}_{1}$ has a closed graph in $\mathcal{E}_{1} \times \mathcal{E}_{1}$.

Then, this semigroup possesses a global attractor $\mathcal{A} \subset \mathcal{B}$ which is generated by all complete bounded trajectories

$$
\mathcal{A}=\left.\mathcal{K}\right|_{t=0},
$$

where $\mathcal{K} \subset L^{\infty}\left(\mathbb{R}, \mathcal{E}_{1}\right)$ is a set of functions $u: \mathbb{R} \rightarrow \mathcal{E}_{1}$ such that $S(t) u(h)=u(t+h)$ for all $h \in \mathbb{R}$ and $t \geq 0$.

For the proof of this proposition, see Ref. 24.

We are now ready to state and prove the main result of this section.

Theorem 3.4. Suppose that the conditions of Theorem 1.1 are satisfied. Then, the semigroup $S(t)$ associated with problem (1.1) possesses a global attractor $\mathcal{A}$ in the phase space $\mathcal{E}_{1}$.

Proof. Indeed, the second assumption of Proposition 3.3 is an immediate corollary of Proposition 2.2, so we only need to check the first one. To this end, we split a solution $u$ of equation (1.1) in a sum $u(t):=v(t)+w(t)$, where the function $v$ solves the linear problem

$$
\left\{\begin{array}{l}
\partial_{t}^{2} v-\gamma \partial_{t} \Delta_{x} v-\Delta_{x} v=h,\left.v\right|_{\partial \Omega}=0 \\
\left.\xi_{v}\right|_{t=0}=\left.\xi_{u}\right|_{t=0}
\end{array}\right.
$$


and the reminder $w$ satisfies

$$
\left\{\begin{array}{l}
\partial_{t}^{2} w-\gamma \partial_{t} \Delta_{x} w-\Delta_{x} w=-f(u) \partial_{t} u-g(u),\left.\quad w\right|_{\partial \Omega}=0, \\
\left.\xi_{w}\right|_{t=0}=0 .
\end{array}\right.
$$

Moreover, without loss of generality, we may assume that $g(0)=0$. The properties of functions $v$ and $w$ are collected in the following two lemmas.

Lemma 3.5. Let the above assumptions hold and let $H=H(x) \in H^{2}(\Omega) \cap H_{0}^{1}(\Omega)$ be the solution of the problem

$$
-\Delta_{x} H=h, x \in \Omega ;\left.u\right|_{\partial \Omega}=0
$$

Then, the following estimate holds:

$$
\left\|\xi_{v}(t)-\xi_{H}\right\|_{\mathcal{E}_{1}} \leq C\left(\left\|\xi_{u}(0)\right\|_{\mathcal{E}_{1}}+\|h\|_{L^{2}}\right) e^{-\alpha t},
$$

where $\xi_{H}=(H, 0)$ and positive constants $C$ and $\alpha$ are independent of $u$.

Proof of the Lemma. Indeed, introducing the new variable $\tilde{v}(t):=v(t)-H$, we reduce (3.5) to the homogeneous form

$$
\left\{\begin{array}{l}
\partial_{t}^{2} \tilde{v}-\gamma \partial_{t} \Delta_{x} \tilde{v}-\Delta_{x} \tilde{v}=0,\left.\tilde{v}\right|_{\partial \Omega}=0, \\
\left.\xi_{\tilde{v}}\right|_{t=0}=\left.\xi_{u}\right|_{t=0}-\xi_{H}
\end{array}\right.
$$

Multiplying this equation by $\partial_{t} \Delta_{x} \tilde{v}+\beta \Delta_{x} \tilde{v}$, where $\beta$ is a small positive parameter, and arguing in a standard way, we derive that

$$
\left\|\xi_{\tilde{v}}(t)\right\|_{\mathcal{E}_{1}}^{2} \leq C\left\|\xi_{\tilde{v}}(0)\right\|_{\mathcal{E}_{1}}^{2} e^{-\alpha t}
$$

for some positive $C$ and $\alpha$, see, e.g., Refs. 2 and 25 as well as the proof of Lemma 3.6. The desired estimate (3.7) is an immediate corollary of this estimate and Lemma 3.5 is proved.

Thus, we have proved that the $v$ component of the solution $u$ converges exponentially to a single function $H \in H^{2}(\Omega)$ which is independent of time and the initial data. The next lemma shows that the $w$ component is more regular.

\section{Lemma 3.6. Let the above assumptions hold and let}

$$
\mathcal{E}_{2}:=\left[H^{3}(\Omega) \cap\left\{\left.u\right|_{\partial \Omega}=\left.\Delta_{x} u\right|_{\partial \Omega}=0\right\}\right] \times\left[H^{2}(\Omega) \cap H_{0}^{1}(\Omega)\right] .
$$

Then, the solution $w$ of problem (3.6) belongs to $\mathcal{E}_{2}$ for all $t \geq 0$ and the following estimate holds:

$$
\left\|\xi_{w}(t)\right\|_{\mathcal{E}_{2}} \leq Q\left(\left\|\xi_{u}(0)\right\|_{\mathcal{E}_{1}}\right) e^{-\alpha t}+Q\left(\|h\|_{L^{2}}\right)
$$

for some positive constant $\alpha$ and monotone function $Q$ which are independent of $u$.

Proof of the Lemma. We give below only the formal derivation of estimate (3.11) which can be justified, e.g., using the Galerkin approximations. First, due to the assumption $g(0)=0$, it follows from Eq. (3.6) that at least formally $\left.\Delta_{x} w\right|_{\partial \Omega}=0$, so we may multiply Eq. (3.6) by $\partial_{t} \Delta_{x}^{2} w+\beta \Delta_{x}^{2} w$ and do integration by parts. This gives

$$
\begin{aligned}
\frac{d}{d t}\left(\frac{1}{2}\left\|\xi_{w}\right\|_{\mathcal{E}_{2}}^{2}+\beta\left(\nabla_{x} \Delta_{x} w\right.\right. & \left.\left., \nabla_{x} \partial_{t} w\right)+\frac{\gamma \beta}{2}\left\|\nabla_{x} \Delta_{x} w\right\|_{L^{2}}^{2}\right)+\beta\left\|\nabla_{x} \Delta_{x} w\right\|_{L^{2}}^{2}-\beta\left\|\partial_{t} \Delta_{x} w\right\|_{L^{2}}^{2}+ \\
& +\gamma\left\|\partial_{t} \nabla_{x} \Delta_{x} w\right\|_{L^{2}}^{2}=\left(\nabla_{x}\left(f(u) \partial_{t} u+g(u)\right), \nabla_{x}\left(\partial_{t} \Delta_{x} w+\beta \Delta_{x} w\right)\right) .
\end{aligned}
$$

Fixing $\beta>0$ small enough and using the notation

$$
E_{2}(w):=\frac{1}{2}\left\|\xi_{w}\right\|_{\mathcal{E}_{2}}^{2}+\beta\left(\nabla_{x} \Delta_{x} w, \nabla_{x} \partial_{t} w\right)+\frac{\gamma \beta}{2}\left\|\nabla_{x} \Delta_{x} w\right\|_{L^{2}}^{2},
$$

we see that, on the one hand,

$$
C_{1}\left\|\xi_{w}\right\|_{\mathcal{E}_{2}}^{2} \leq E_{2}(w) \leq C_{2}\left\|\xi_{w}\right\|_{\mathcal{E}_{2}}^{2}
$$


for some positive constants $C_{1}$ and $C_{2}$. On the other hand, Eq. (3.12) implies that

$$
\frac{d}{d t} E_{2}(w)+\alpha E_{2}(w) \leq C\left(\left\|f(u) \partial_{t} u\right\|_{H^{1}}^{2}+\|g(u)\|_{H^{1}}^{2}\right)
$$

for some positive constants $C$ and $\alpha$. Finally, using the embedding $H^{2}(\Omega) \subset C(\Omega)$ and growth restrictions (1.2), we estimate the right-hand side of (3.14) as follows:

$$
\left\|f(u) \partial_{t} u\right\|_{H^{1}}^{2}+\|g(u)\|_{H^{1}}^{2} \leq C\left(\|u\|_{H^{2}}^{p+q+1}+1\right)\left(\left\|\partial_{t} \nabla_{x} u\right\|_{L^{2}}^{2}+1\right) .
$$

Applying the Gronwall inequality to (3.14) and using (3.15) and (3.13) together with the dissipative estimate (2.24), we derive the desired estimate (3.11) and finish the proof of Lemma 3.6.

It is not difficult now to finish the proof of the theorem. Indeed, Lemmas 3.5 and 3.6 show that the set

$$
\mathcal{B}:=\xi_{H}+\left\{w \in \mathcal{E}_{2},\left\|\xi_{w}\right\|_{\mathcal{E}_{2}} \leq R\right\}
$$

will be a compact attracting set for the semigroup $S(t)$ generated by problem (1.1) if $R$ is large enough. Thus, all assumptions of Proposition 3.3 are verified and the theorem is proved.

Remark 3.7. As already it was mentioned in the Introduction, we do not know how to deduce the basic dissipative estimate for the weak solutions of problem (1.1) in the phase space $\mathcal{E}$ in the case when condition (1.6) is violated. However, as follows from Theorem 2.1, we have such an estimate in the intermediate space $\left[H^{2}(\Omega) \cap H^{1}(\Omega)\right] \times L^{2}(\Omega)$ which is in a sense natural for strongly damped wave equations, see Refs. 11 and 22. Actually, the problem is well posed in this space and the above developed attractor theory can be extended to this phase space as well.

\section{ACKNOWLEDGMENTS}

The work of V. K. Kalantarov was supported in part by The Scientific and Research Council of Turkey, Grant No. 112T934.

${ }^{1}$ R. Arima and Y. Hasegawa, “On global solutions for mixed problem of a semi-linear differential equation,” Proc. Jpn. Acad. 39, 721-725 (1963).

2 A. Babin and M. Vishik, Attractors of Evolution Equations (Amsterdam, North-Holland, 1992).

${ }^{3}$ V. Barbu, I. Lasiecka, and M. A. Rammaha, "On nonlinear wave equations with degenerate damping and source terms," Trans. Am. Math. Soc. 357(7), 2571-2611 (2005).

${ }^{4}$ V. Belykh, N. Pedersen, and O. Soerensen, "Shunted-Josephson-junction model. I. The autonomous case," Phys. Rev. B 16(11), 4853-4859 (1977)

${ }^{5}$ A. Carvalho and J. Cholewa, “Attractors for strongly damped wave equations with critical nonlinearities," Pac. J. Math. 207, 287-310 (2002).

${ }^{6}$ A. Carvalho, J. Cholewa, and T. Dlotko, "Strongly damped wave problems: Bootstrapping and regularity of solutions," J. Differ. Equations 244(9), 2310-2333 (2008).

${ }^{7}$ F. Dell' Oro and V. Pata, "Strongly damped wave equations with critical nonlinearities," Nonlinear Anal. 75(14), 5723-5735 (2012).

${ }^{8}$ J. Ghidaglia and A. Marzocchi, "Longtime behaviour of strongly damped wave equations, global attractors and their dimension,” SIAM J. Math. Anal. 22, 879-895 (1991).

${ }^{9}$ V. K. Kalantarov, "Attractors for some nonlinear problems of mathematical physics," Zap. Nauchn. Sem. Leningrad. Otdel. Mat. Inst. Steklov, (LOMI) 152, 50-54 (1986).

${ }^{10}$ V. K. Kalantarov, "Global behavior of solutions of nonlinear equations of mathematical physics of classical and non-classical types," Ph.D. Thesis (St.Petersburg Department of Steklov Math. Inst., St.Petersburg, 1988).

${ }^{11}$ V. Kalantarov and S. Zelik, "Finite-dimensional attractors for the quasi-linear strongly-damped wave equation," J. Differ Equations 247, 1120-1155 (2009).

12 A. Khanmamedov, "Long-time behaviour of wave equations with nonlinear interior damping," Discrete Contin. Dyn. Syst. 21(4), 1185-1198 (2008).

${ }^{13}$ A. Khanmamedov, "Global attractors for 2-D wave equations with displacement dependent damping," Math. Methods Appl. Sci. 33, 177-187 (2010).

${ }^{14}$ A. Khanmamedov, "Global attractors for strongly damped wave equations with displacement dependent damping and nonlinear source term of critical exponent," Discrete Contin. Dyn. Syst. 31(1), 119-138 (2011).

${ }^{15}$ A. Khanmamedov, "Strongly damped wave equation with exponential nonlinearities," J. Math. Anal. Appl. 419, 663-687 (2014); e-print arXiv:1212.2180. 
${ }^{16}$ P. Lomdahl, O. Soerensen, and P. Christiansen, “Soliton excitations in Josephson tunnel junctions,” Phys. Rev. B 25, 5337-5348 (1982).

${ }^{17} \mathrm{H}$. Li, S. Zhou, and F. Yin, "Global periodic attractor for strongly damped wave equations with time-periodic driving force," J. Math. Phys. 45, 3462-3467 (2004).

${ }^{18}$ P. Massatt, "Limiting behavior for strongly damped nonlinear wave equations," J. Differ. Equations 48, 334-349 (1983).

${ }^{19}$ V. Pata and M. Squassina, "On the strongly damped wave equation," Commun. Math. Phys. 253, 511-533 (2005).

${ }^{20}$ U. Poulsen, "On the $\cos \phi$ term in Josephson's expression for the tunelling current," Rev. Phys. Appl. 9, 41-44 (1974)

${ }^{21}$ M. Rammaha and T. Strei, "Global existence and nonexistence for nonlinear wave equations with damping and source terms," Trans. Am. Math. Soc. 354(9), 3621-3637 (2002).

22 V. Pata and S. Zelik, "Smooth attractors for strongly damped wave equations," Nonlinearity 19, 1495-1506 (2006).

${ }^{23}$ V. Pata and S. Zelik, "Global and exponential attractors for 3-D wave equations with displacement dependent damping," Math. Methods Appl. Sci. 29, 1291-1306 (2006).

${ }^{24}$ V. Pata and S. Zelik, "A result on the existence of global attractors for semigroups of closed operators," Commun. Pure Appl. Anal. 6(2), 481-486 (2007).

${ }^{25}$ R. Temam, Infinite-Dimensional Dynamical Systems in Mechanics and Physics (Springer-Verlag, New York, 1988).

${ }^{26}$ M. Yang and C. Sun, "Dynamics of strongly damped wave equations in locally uniform spaces: Attractors and asymptotic regularity," Trans. Am. Math. Soc. 361(2), 1069-1101 (2009).

${ }^{27}$ G. Webb, "Existence and asymptotic behavior for a strongly damped nonlinear wave equation," Can. J. Math. 32, 631-643 (1980).

${ }^{28}$ S. Zhou and X. Fan, "Kernel sections for non-autonomous strongly damped wave equations," J. Math. Anal. Appl. 275(2), 850-869 (2002). 